\title{
Step scaling and the Yang-Mills gradient flow
}

\author{
Martin Lüscher \\ CERN, Physics Department, \\ 1211 Geneva 23, Switzerland \\ E-mail: luscher@mail.cern.ch
}

ABSTRACT: The use of the Yang-Mills gradient flow in step-scaling studies of lattice QCD is expected to lead to results of unprecedented precision. Step scaling is usually based on the Schrödinger functional, where time ranges over an interval $[0, T]$ and all fields satisfy Dirichlet boundary conditions at time 0 and $T$. In these calculations, potentially important sources of systematic errors are boundary lattice effects and the infamous topology-freezing problem. The latter is here shown to be absent if Neumann instead of Dirichlet boundary conditions are imposed on the gauge field at time 0 . Moreover, the expectation values of gauge-invariant local fields at positive flow time (and of other well localized observables) that reside in the center of the space-time volume are found to be largely insensitive to the boundary lattice effects.

KEYwords: Lattice QCD, Renormalization Group, Lattice Quantum Field Theory

ArXiv EPRINT: 1404.5930 


\section{Contents}

1 Introduction $\quad 2$

2 QCD with open-SF boundary conditions 3

2.1 Continuum theory 3

2.2 Lattice formulation 3

2.3 Lattice action 4

2.4 Stability at weak coupling 5

3 Perturbation theory 5

3.1 Fourier representation 6

$\begin{array}{ll}3.2 \text { Gauge fixing } & 6\end{array}$

$\begin{array}{ll}3.3 \text { Gauge-field propagator } & 7\end{array}$

4 The gradient-flow coupling $\quad 8$

4.1 Flow equation 8

4.2 Solution of the flow equation to leading order in $g_{0} \quad 8$

4.3 Running coupling $\quad 9$

5 Topology-freezing and autocorrelation times $\quad 10$

$\begin{array}{lll}5.1 & \text { Definition of the topological charge } Q & 10\end{array}$

$\begin{array}{lll}5.2 & \text { Topology-freezing with SF boundary conditions } & 10\end{array}$

$\begin{array}{ll}5.3 & \text { Autocorrelation times from large to small volumes } \\ 5.42\end{array}$

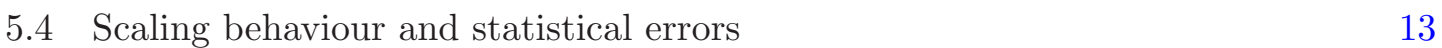

6 Boundary lattice effects $\quad 14$

$\begin{array}{ll}\text { 6.1 How large are the boundary lattice effects? } & 14\end{array}$

$\begin{array}{lll}6.2 & \text { Finite-size effects and the large-volume limit } & 14\end{array}$

$\begin{array}{lll}6.3 & \text { Synthesis } & 16\end{array}$

$\begin{array}{lll}7 & \text { Concluding remarks } & 17\end{array}$

$\begin{array}{lr}\text { A Notational conventions } & 18\end{array}$

$\begin{array}{ll}\text { B Normalization of the gradient-flow coupling } & \mathbf{1 8}\end{array}$

$\begin{array}{ll}\text { B.1 Open-SF boundary conditions } & 19\end{array}$

$\begin{array}{lll}\text { B.2 SF boundary conditions } & 19\end{array}$

$\begin{array}{lll}\text { B.3 Explicit form of the gauge-field propagator } & 19\end{array}$ 


\section{Introduction}

In numerical lattice field theory, step scaling refers to a finite-size scaling technique that allows the physics at high energies to be related to the characteristic low energy scales of the theory [1]. While no uncontrolled approximations need to be made in these calculations, the precision of the results that can be reached in practice depends on many details. In particular, the boundary conditions in finite volume and the observables should be such that the systematic and statistical errors are minimized.

Using the Yang-Mills gradient flow [2]-[4], many observables can be constructed that are expected to be well suited for step-scaling studies. The expectation value of the square of the gauge-field tensor at positive flow time, for example, can serve as running coupling in finite volume [5]-[10]. Another motivation for considering such observables derives from their small flow-time expansion in local fields $[3,11]-[15]$. Over a wide range of flow time, the coefficients in these expansions may conceivably be computed using an adapted stepscaling technique. Representations of local fields would then be obtained, where the field renormalization and Symanzik-improvement are greatly simplified.

In lattice QCD, step scaling is usually based on the Schrödinger functional [16, 17]. There are good reasons for this choice, among them the absence of zero-modes in perturbation theory, the renormalizability of the Schrödinger functional and the fact that the theory can be probed by varying the boundary values of the gauge field. On the other hand, the boundaries of the lattice in the time direction are a source of additional lattice effects. Moreover, near the continuum limit, simulations of the Schrödinger functional tend to get trapped in one of the emergent sectors of fixed topological charge of the gauge field.

Until recently [18], topology-freezing was not thought to be an issue in step-scaling studies. In small space-time volumes, where most of the step scaling is performed, the non-trivial topological sectors are in fact strongly suppressed. The width of the (unbiased) charge distribution however grows rapidly towards the non-perturbative large-volume regime. A proper sampling of the charge sectors is then required, or a way of bypassing the problem (such as restricting the theory to the trivial sector [18]) must be found. Resolving this technical issue is particularly urgent when observables defined at positive gradient-flow time are used, since these can be rather sensitive to autocorrelations in simulation time [19].

In this paper, a modification of the Schrödinger functional is considered, where open (Neumann) boundary conditions are imposed on the gauge field at one of the space-time boundaries and Dirichlet boundary conditions at the opposite boundary. With this choice, the renormalizability of the theory is preserved, the perturbation expansion in powers of the gauge coupling remains regular and the topology-freezing problem is avoided, because the topological charge can freely flow in and out of the volume through the open boundary [19]. The volume-dependence of the expectation values of observables localized in the center of the space-time volume is then examined and shown to be small in the kinematical situations of interest. In particular, such quantities are practically unaffected by boundary lattice effects. Both limitations of the Schrödinger functional setup can thus be overcome with the suggested change of boundary conditions and if suitable local observables (such as the ones obtained with the gradient flow) are used. 


\section{QCD with open-SF boundary conditions}

The proposed modification of the Schrödinger functional $[16,17]$ is rather modest and only concerns the gauge field. In particular, the boundary conditions imposed on the quark fields are left unchanged and need not be discussed here.

\subsection{Continuum theory}

As in the case of the Schrödinger functional, the theory is set up on a four-dimensional box with Euclidean metric, time-like extent $T$ and size $L$ in the space directions. Periodic boundary conditions are chosen in space so that the space-time manifold has two boundaries, one at, say, time $x_{0}=0$ and the other at $x_{0}=T$.

For simplicity, only homogeneous boundary conditions are considered here, where the gauge potential $A_{\mu}(x)$ is required to satisfy

$$
\begin{array}{ll}
\left.F_{0 k}(x)\right|_{x_{0}=0}=0, & F_{\mu \nu}(x)=\partial_{\mu} A_{\nu}-\partial_{\nu} A_{\mu}+\left[A_{\mu}, A_{\nu}\right], \\
\left.A_{k}(x)\right|_{x_{0}=T}=0, & k=1,2,3
\end{array}
$$

(the notational conventions are summarized in appendix A). The only difference with respect to the Schrödinger functional is the choice of Neumann rather than Dirichlet boundary conditions at time 0 . Neumann boundary conditions are also referred to as open boundary conditions and the term "open-SF boundary conditions" is, in the following, reserved for the combination (2.1), (2.2) of boundary conditions. For brevity, homogenous Dirichlet boundary conditions at both time 0 and time $T$ will be referred to as "SF boundary conditions".

With open-SF boundary conditions, the gauge potential remains unconstrained at $x_{0}=0$ and only its (gauge-covariant) time derivative must vanish. As a consequence, the topological charge of the gauge field is no longer quantized and can freely flow in and out of the space-time volume through the boundary [19]. The renormalizability of the theory, on the other hand, is preserved, since there are no candidate boundary counterterms of dimension 3 with the required symmetry properties $[16,17,19]$.

\subsection{Lattice formulation}

In the lattice theory, space-time is replaced by a hypercubic lattice of points $x$ with integer Cartesian coordinates $x_{0}, x_{1}, x_{2}, x_{3}$ in the range

$$
0 \leq x_{0} \leq T, \quad 0 \leq x_{k}<L \quad(k=1,2,3)
$$

For notational convenience, the lattice sizes $T, L$ and all other dimensionful quantities are given in units of the lattice spacing $a$. Periodic boundary conditions in space imply that any two points $x$ and $x+\hat{k} \bmod L$ (where $\hat{\mu}$ denotes the unit vector in direction $\mu$ ) are considered to be nearest neighbors.

As usual the gauge field is represented by a field of matrices $U(x, \mu) \in \mathrm{SU}(N)$ on the links $(x, x+\hat{\mu})$ of the lattice. No field variables are assigned to the links that "stick out of 

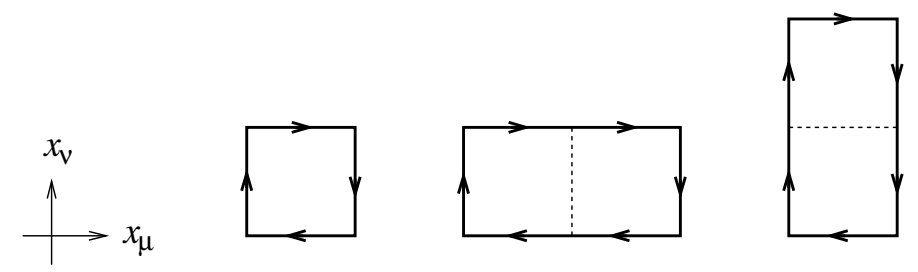

Figure 1. Plaquette and planar double-plaquette loops in a $(\mu, \nu)$-plane of the lattice. Loops differing by their orientation are considered to be different.

the lattice", i.e. $\mu=0,1,2,3$ if $0 \leq x_{0}<T$ and $\mu=1,2,3$ if $x_{0}=T$. Imposing open-SF boundary conditions amounts to setting

$$
\left.U(x, k)\right|_{x_{0}=T}=1, \quad k=1,2,3,
$$

while all other field variables are left unconstrained and are integrated over in the QCD functional integral. In particular, the boundary condition (2.1) does not need to be enforced on the lattice, since it emerges dynamically when the continuum limit is taken [19].

\subsection{Lattice action}

Let $\mathcal{S}_{0}$ and $\mathcal{S}_{1}$ be the sets of oriented plaquette and double-plaquette loops on the lattice (see figure 1). Many popular lattice actions for the gauge field are of the general form [20]-[22]

$$
S_{\mathrm{G}}=\frac{1}{g_{0}^{2}} \sum_{k=0}^{1} c_{k} \sum_{\mathcal{C} \in \mathcal{S}_{k}} w_{k}(\mathcal{C}) \operatorname{tr}\{1-U(\mathcal{C})\}
$$

where $U(\mathcal{C})$ denotes the ordered product of the link variables around the loop $\mathcal{C}$. The weight factors $w_{k}(\mathcal{C})$ differ from unity only near the boundaries of the lattice and will be specified below.

In order to ensure the correct normalization of the bare coupling $g_{0}$, the coefficients $c_{k}$ must be such that

$$
c_{0}+8 c_{1}=1
$$

Moreover, the constraint $c_{0}>0$ is imposed as otherwise there may be fields with lowest action which are not locally pure-gauge configurations [22].

The sum in eq. (2.5) runs over all loops $\mathcal{C}$ that are fully contained in the range $0 \leq$ $x_{0} \leq T$ of time. In addition, the double-plaquette loops shown in figure 2 are included in the sum. The weights of the plaquette loops are then

$$
w_{0}(\mathcal{C})= \begin{cases}\frac{1}{2} c_{\mathrm{G}} & \text { if } \mathcal{C} \text { is a spatial loop at } x_{0}=0, \\ c_{\mathrm{G}}^{\prime} & \text { if } \mathcal{C} \text { has exactly one spatial link at } x_{0}=T, \\ 1 & \text { otherwise },\end{cases}
$$



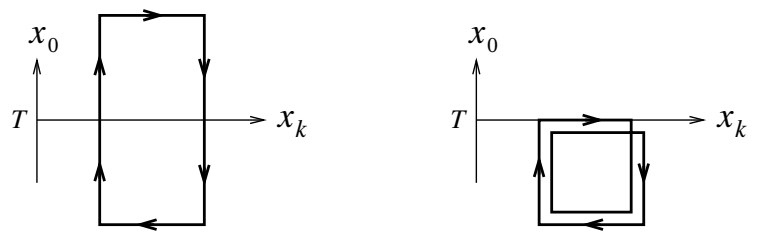

Figure 2. The time-like double-plaquette loops $\mathcal{C}$ that cross the boundary at time $T$ are included in the gauge action $(2.5)$, with weight $w_{1}(\mathcal{C})=1 / 2$ and $U(\mathcal{C})$ set to the product of the link variables around the loop shown on the right.

while those of the double-plaquette loops are given by

$$
w_{1}(\mathcal{C})= \begin{cases}\frac{1}{2} c_{\mathrm{G}} & \text { if } \mathcal{C} \text { is a spatial loop at } x_{0}=0 \\ \frac{1}{2} & \text { if } \mathcal{C} \text { crosses the boundary at } x_{0}=T \text { as in figure } 2 \\ 1 & \text { otherwise }\end{cases}
$$

where $c_{\mathrm{G}}$ and $c_{\mathrm{G}}^{\prime}$ are improvement coefficients that should be tuned so as to cancel the $\mathrm{O}(a)$ boundary lattice effects in on-shell quantities [16]. At tree-level of perturbation theory, this is achieved by setting $c_{\mathrm{G}}=c_{\mathrm{G}}^{\prime}=1 .^{1}$

\subsection{Stability at weak coupling}

At small coupling $g_{0}$, the QCD functional integral is dominated by the gauge-field configurations in the neighborhood of the configurations with lowest action. The latter can be shown to be gauge-equivalent to the classical vacuum configuration, both in the continuum theory and in the $\mathrm{O}(a)$-improved lattice theory [22]. Moreover, the Hessian of the action at the classical vacuum configuration has no zero modes other than the gauge modes (see section 3). With open-SF boundary conditions, the theory is thus practically Gaussian in the weak-coupling domain and is therefore expected to be entirely well-behaved in this regime.

\section{Perturbation theory}

The perturbation expansion of the theory with open-SF boundary conditions does not run into any particular difficulties and actually tends to be slightly simpler than in the case of the Schrödinger functional. In this section, the gauge-field propagator is calculated in the $\mathrm{O}(a)$-improved lattice theory. The result is obtained in a form in which the continuum limit is transparent and that allows the normalization of the gradient-flow coupling to be determined in a few lines (see section 4 ).

\footnotetext{
${ }^{1}$ The form of the action near the boundary at $x_{0}=T$ differs from both choice A and B proposed by Aoki, Frezzotti and Weisz [23]. Choice B is recommended for studies of the Schrödinger functional with a non-trivial background field, while $\mathrm{A}$ is the more convenient choice for perturbation theory in the absence of a background field. The action defined here combines the good properties of A and B without having any obvious disadvantages.
} 


\subsection{Fourier representation}

In perturbation theory, the link variables ${ }^{2}$

$$
U(x, \mu)=\exp \left\{g_{0} A_{\mu}(x)\right\}
$$

are parameterized through a lattice gauge potential $A_{\mu}(x)$. The latter can be decomposed into Fourier modes according to

$$
\begin{aligned}
& A_{0}(x)=\frac{2 i}{T L^{3}} \sum_{p} \sin \left(p_{0} x_{0}+\frac{1}{2} p_{0}\right) \mathrm{e}^{i \mathbf{p x}} \tilde{A}_{0}(p), \\
& A_{k}(x)=\frac{2}{T L^{3}} \sum_{p} \cos \left(p_{0} x_{0}\right) \mathrm{e}^{i \mathbf{p x}+\frac{i}{2} p_{k}} \tilde{A}_{k}(p),
\end{aligned}
$$

where the momentum components run over the values

$$
\begin{aligned}
& p_{0}=\left(n_{0}+\frac{1}{2}\right) \frac{\pi}{T}, \quad n_{0}=0,1, \ldots, T-1, \\
& p_{k}=n_{k} \frac{2 \pi}{L}, \quad n_{k}=0,1, \ldots, L-1, \quad k=1,2,3 .
\end{aligned}
$$

Note that $p=0$ is not contained in the momentum spectrum and that the Fourier representation (3.3) respects the boundary condition (2.4) since $\cos \left(p_{0} T\right)=0$ for all momenta. In view of the reality of the gauge potential in position space, the Fourier components must satisfy

$$
\tilde{A}_{0}^{a}(p)^{*}=-\tilde{A}_{0}^{a}\left(p_{0},-\mathbf{p}\right), \quad \tilde{A}_{k}^{a}(p)^{*}=\tilde{A}_{k}^{a}\left(p_{0},-\mathbf{p}\right),
$$

but are otherwise unconstrained.

Some algebra now shows that the gauge action (2.5) [with $\left.c_{\mathrm{G}}, c_{\mathrm{G}}^{\prime}=1+\mathrm{O}\left(g_{0}^{2}\right)\right]$ is given by

$$
S_{\mathrm{G}}=\frac{1}{2 T L^{3}} \sum_{p} \sum_{\mu, \nu}\left(1-c_{1}\left(\hat{p}_{\mu}^{2}+\hat{p}_{\nu}^{2}\right)\right)\left|\hat{p}_{\mu} \tilde{A}_{\nu}^{a}(p)-\hat{p}_{\nu} \tilde{A}_{\mu}^{a}(p)\right|^{2}+\mathrm{O}\left(g_{0}\right)
$$

to leading order in the gauge coupling, where

$$
\hat{p}_{\mu}=2 \sin \left(\frac{1}{2} p_{\mu}\right)
$$

The Fourier transformation thus diagonalizes the quadratic part of the action. One might consider this to be hardly worth pointing out, but whether the property holds or not actually depends on the exact form of the action near the boundaries of the lattice [23].

\subsection{Gauge fixing}

With the chosen boundary conditions, it suffices to fix the subgroup of gauge transformations $\Lambda(x) \in \mathrm{SU}(N)$ satisfying

$$
\left.\Lambda(x)\right|_{x_{0}=T}=1 .
$$

\footnotetext{
${ }^{2}$ For notational convenience, the same symbol is used for the gauge potential as in the continuum theory. Notice that the gauge potential is scaled by the coupling $g_{0}$ in perturbation theory.
} 
The global gauge transformations then remain a symmetry of the gauge-fixed theory. A possible choice of the gauge-fixing term is

$$
\begin{aligned}
S_{\mathrm{gf}} & =\frac{1}{2} \lambda_{0}(ð A, \partial A), \\
(ð A)(x) & =\sum_{\mu}\left(1+c_{1} \partial_{\mu}^{*} \partial_{\mu}\right) \partial_{\mu}^{*} A_{\mu}(x),
\end{aligned}
$$

where $\partial_{\mu}$ and $\partial_{\mu}^{*}$ denote the standard forward and backward difference operators, $\lambda_{0}>0$ is the gauge-fixing parameter and the terms proportional to $c_{1}$ are included in order to simplify the expression for the gauge-field propagator (see appendix B). The scalar product in eq. (3.10),

$$
(\omega, \nu)=\sum_{x_{0}=0}^{T} \sum_{\mathbf{x}} \omega^{a}(x) \nu^{a}(x),
$$

is the one in the space of infinitesimal gauge transformations (the primed summation symbol indicates that the terminal summands are counted with weight $1 / 2$ ).

Near the boundaries of the lattice, the derivatives in eq. (3.11) require some further specification, since the gauge potential $A_{\mu}(x)$ is only defined on the links $(x, x+\hat{\mu})$ in the range $[0, T]$ of time. A simple prescription for the boundary terms amounts to extending the potential to all points $x$ with integer coordinates through its Fourier representation (3.2), (3.3) so that

$$
(ð A)(x)=\frac{2 i}{T L^{3}} \sum_{p} \sum_{\mu} \cos \left(p_{0} x_{0}\right) \mathrm{e}^{i \mathbf{p x}}\left(\hat{p}_{\mu}-c_{1} \hat{p}_{\mu}^{3}\right) \tilde{A}_{\mu}(p) .
$$

For the gauge-fixed action the formula

$$
\begin{aligned}
S_{\mathrm{G}}+S_{\mathrm{gf}}= & \frac{1}{T L^{3}} \sum_{p} \sum_{\mu, \nu} \tilde{A}_{\mu}^{a}(p)^{*} \Delta_{\mu \nu}(p) \tilde{A}_{\nu}^{a}(p)+\mathrm{O}\left(g_{0}\right), \\
\Delta_{\mu \nu}(p)= & \delta_{\mu \nu} \hat{p}^{2}-\hat{p}_{\mu} \hat{p}_{\nu}-c_{1}\left\{\delta_{\mu \nu}\left[\hat{p}^{4}+\frac{1}{2} \hat{p}^{2}\left(\hat{p}_{\mu}^{2}+\hat{p}_{\nu}^{2}\right)\right]-\hat{p}_{\mu}^{3} \hat{p}_{\nu}-\hat{p}_{\mu} \hat{p}_{\nu}^{3}\right\} \\
& +\lambda_{0}\left(\hat{p}_{\mu}-c_{1} \hat{p}_{\mu}^{3}\right)\left(\hat{p}_{\nu}-c_{1} \hat{p}_{\nu}^{3}\right), \\
\hat{p}^{2}= & \sum_{\mu} \hat{p}_{\mu}^{2}, \quad \hat{p}^{4}=\sum_{\mu} \hat{p}_{\mu}^{4},
\end{aligned}
$$

is then obtained. The associated Faddeev-Popov action is diagonal in momentum space too, the inverse propagator being $\Delta(p)=\hat{p}^{2}-c_{1} \hat{p}^{4}$ in this case.

\subsection{Gauge-field propagator}

The normalization condition (2.6) and the requirement that the coefficient $c_{0}$ must be positive imply the strict positivity of the matrix $\Delta_{\mu \nu}(p)$ for all momenta $p$ summed over in eq. (3.14). In particular, the gauge-fixed action has no zero-modes and the gauge-field propagator in momentum space is given by

$$
\left\langle\tilde{A}_{\mu}^{a}(p) \tilde{A}_{\nu}^{b}(q)^{*}\right\rangle=\frac{1}{2} T L^{3} \delta^{a b} \delta_{p q} D_{\mu \nu}(p)+\mathrm{O}\left(g_{0}\right),
$$


where $D_{\mu \nu}(p)$ denotes the (matrix) inverse of $\Delta_{\mu \nu}(p)$. Recalling the Fourier representation (3.2), (3.3) of the gauge potential, it is straightforward to pass from here to the propagator in position space.

\section{The gradient-flow coupling}

Apart from some minor technical details, the definition of the gradient-flow coupling in the theory with open-SF boundary conditions is the same as in the case of periodic and SF boundary conditions considered in refs. $[5,6]$ and $[7,8]$, respectively. All formulae in this section refer to the lattice theory, but their form in the continuum limit should be quite obvious (see refs. $[2,15]$ for an introduction to the subject).

\subsection{Flow equation}

The Yang-Mills gradient flow evolves the gauge field as a function of a parameter $t \geq 0$, which is referred to as the flow time. On the lattice, the link variables $V_{t}(x, \mu)$ at flow time $t$ are determined by the boundary condition

$$
\left.V_{t}(x, \mu)\right|_{t=0}=U(x, \mu)
$$

and the first-order evolution equation

$$
\partial_{t} V_{t}(x, \mu) V_{t}(x, \mu)^{-1}=-w_{x, \mu} g_{0}^{2}\left(\partial_{x, \mu}^{a} S_{\mathrm{w}}\right)\left(V_{t}\right) T^{a},
$$

where $S_{\mathrm{w}}$ denotes the Wilson action, i.e. the gauge action (2.5) with $c_{0}=c_{\mathrm{G}}=c_{\mathrm{G}}^{\prime}=1$ and $c_{1}=0$. The gradient of the action on the right of the flow equation is defined in appendix A and the weight factor $w_{x, \mu}$ is given by

$$
w_{x, \mu}= \begin{cases}2 & \text { if } x_{0}=0 \text { and } \mu>0 \\ 0 & \text { if } x_{0}=T \text { and } \mu>0 \\ 1 & \text { otherwise }\end{cases}
$$

Assigning weight 2 to the spatial links at $x_{0}=0$ ensures that the gradient flow does not generate $\mathrm{O}(a)$ lattice effects at positive flow time [24]. At $x_{0}=T$, on the other hand, the weight factor is set to zero so that the boundary values of the gauge field are preserved.

\subsection{Solution of the flow equation to leading order in $g_{0}$}

In perturbation theory, the link variables

$$
V_{t}(x, \mu)=\exp \left\{g_{0} B_{\mu}(t, x)\right\},\left.\quad B_{\mu}(t, x)\right|_{t=0}=A_{\mu}(x),
$$

are parameterized by a $t$-dependent gauge potential $B_{\mu}(t, x)$. Furthermore, a gaugedamping term must be added on the right of the flow equation (4.2), as otherwise the gauge modes of the field are not guaranteed to remain small in the course of the flow-time evolution. 
A possible choice of the gauge-damping term is

$$
\begin{aligned}
& \alpha_{0}\left\{V_{t}(x, \mu) \omega_{t}(x+\hat{\mu}) V_{t}(x, \mu)^{-1}-\omega_{t}(x)\right\}, \\
& \omega_{t}(x)=g_{0} \sum_{\nu} \partial_{\nu}^{*} B_{\nu}(t, x),
\end{aligned}
$$

where $\alpha_{0}$ (the "gauge-damping parameter") can be set to any positive value. ${ }^{3}$ As in the continuum theory [2], the structure of the gauge-damping term is such that it can be removed by a $t$-dependent gauge transformation. Gauge-invariant combinations of the link variables at fixed flow time are therefore not affected by the inclusion of the term in the flow equation.

At tree-level of perturbation theory, the flow equation is linear and can be solved analytically by passing to momentum space. Explicitly, the Fourier components of the gauge potential $B_{\mu}(t, x)$ are given by

$$
\tilde{B}_{\mu}(t, p)=\sum_{\nu}\left\{\frac{\mathrm{e}^{-t \hat{p}^{2}}}{\hat{p}^{2}}\left(\hat{p}^{2} \delta_{\mu \nu}-\hat{p}_{\mu} \hat{p}_{\nu}\right) \tilde{A}_{\nu}(p)+\frac{\mathrm{e}^{-\alpha_{0} t \hat{p}^{2}}}{\hat{p}^{2}} \hat{p}_{\mu} \hat{p}_{\nu} \tilde{A}_{\nu}(p)\right\}+\mathrm{O}\left(g_{0}\right)
$$

to leading order in the gauge coupling.

\subsection{Running coupling}

Let $G_{\mu \nu}(t, x)$ be the standard clover lattice expression for the gauge-field tensor at flow time $t$ (see ref. [2], for example). The expectation value of its square,

$$
E(t, x)=\frac{1}{4} \sum_{\mu, \nu} G_{\mu \nu}^{a}(t, x) G_{\mu \nu}^{a}(t, x),
$$

does not require renormalization and has a regular perturbation expansion in powers of $g_{0}^{2}$ without constant term $[2,3]$. In infinite volume and at vanishing quark masses, the dimensionless combination

$$
\bar{g}_{\infty}^{2}=k_{\infty}\left\{t^{2}\langle E(t, x)\rangle\right\}_{\sqrt{8 t}=r},
$$

with the normalization constant $k_{\infty}$ chosen such that

$$
\bar{g}_{\infty}^{2}=g_{0}^{2}+\mathrm{O}\left(g_{0}^{4}\right)
$$

has therefore all the properties of a renormalized coupling that runs with the smoothing range $r$ of the gradient flow.

Renormalized couplings can be defined in exactly the same way in finite volume, but in order to obtain a coupling that runs with $L$, the ratios $T / L, x_{0} / L$ and $r / L$ must be set to some fixed values (as before the quark masses are assumed to vanish). In this paper the coupling is taken to be

$$
\bar{g}^{2}=k\left\{t^{2}\langle E(t, x)\rangle\right\}_{T=L, x_{0}=L / 2, \sqrt{8 t}=c L},
$$

\footnotetext{
${ }^{3}$ At the boundaries of the lattice, the derivatives $\partial_{0}^{*} B_{0}(t, x)$ in eq. (4.6) assume the gauge potential $B_{0}(t, x)$ to be defined at all integer times $x_{0}$ through its Fourier representation. This amounts to setting $\partial_{0}^{*} B_{0}(t, x)=2 B_{0}(t, x)$ at $x_{0}=0$ and $\partial_{0}^{*} B_{0}(t, x)=0$ at $x_{0}=T$.
} 
where $c$ is a dimensionless parameter that is left unspecified for the moment. Some numerical studies of the theory with SF boundary conditions suggest that the statistical variance and lattice-spacing-dependence of the coupling are both reasonably small on the accessible lattices if $c$ is in the range $0.3-0.5[7,8]$. The normalization constant $k$ is again determined by requiring $\bar{g}^{2}$ to coincide with $g_{0}^{2}$ in the weak-coupling limit (see appendix B).

\section{Topology-freezing and autocorrelation times}

On lattices with periodic boundary conditions, the space of gauge fields is connected, but effectively divides into sectors of fixed topological charge close to the continuum limit, with the field space "between the sectors" being suppressed in the functional integral by a large power of the lattice spacing $[2,25]$. Simulations of QCD tend to get trapped in the emerging sectors and may consequently lead to biased results.

The issue has been extensively studied in the past (see refs. [26-28], in particular), and the situation is not fundamentally different if SF boundary conditions are chosen. The topological sectors exist in this case too [16] and so does the topology-freezing problem [18].

\subsection{Definition of the topological charge $Q$}

Since the topological sectors are strictly separated from each other only in the continuum limit, the definition of the topological charge on the lattice requires some choices to be made. As shown in ref. [2], the emergence of the sectors can be quantitatively understood by transforming the QCD functional integral to an integral over the gauge field at gradient-flow time $t>0$. A straightforward discretization of the topological density then leads to the definition

$$
Q=\frac{1}{32 \pi^{2}} \sum_{x} \sum_{\mu, \nu}^{*} G_{\mu \nu}^{a}(t, x) G_{\mu \nu}^{a}(t, x)
$$

which is automatically consistent with the division of the field space in the continuum limit if $t$ is set to some fixed value in physical units. Moreover, the moments $\left\langle Q^{n}\right\rangle$ of the charge distribution do not require renormalization and can be shown to be independent of $t$ up to lattice effects of order $a^{2}$ [2]. In this section, $t$ is scaled with $L$ such that $\sqrt{8 t}=0.3 \times L$, but other reasonable choices of $t$ would make no difference in the following.

When SF or open-SF boundary conditions are imposed, the definition (5.1) needs to be complemented with a prescription of how exactly the charge density is defined at time 0 and $T$. In the continuum theory, the density vanishes at these times and the boundary points may therefore just as well be excluded from the sum over $x$ in eq. (5.1) (for reasons given later, further time-slices near the open boundary will be omitted when studying the autocorrelations of $Q$ ).

\subsection{Topology-freezing with SF boundary conditions}

For illustration, it may be worth considering a sample simulation of the Schrödinger functional, where the topological charge of the gauge field is practically unchanged for very long periods of simulation time (see figure 3 ). In this run, the $\mathrm{O}(a)$-improved theory $[29,30]$ with 


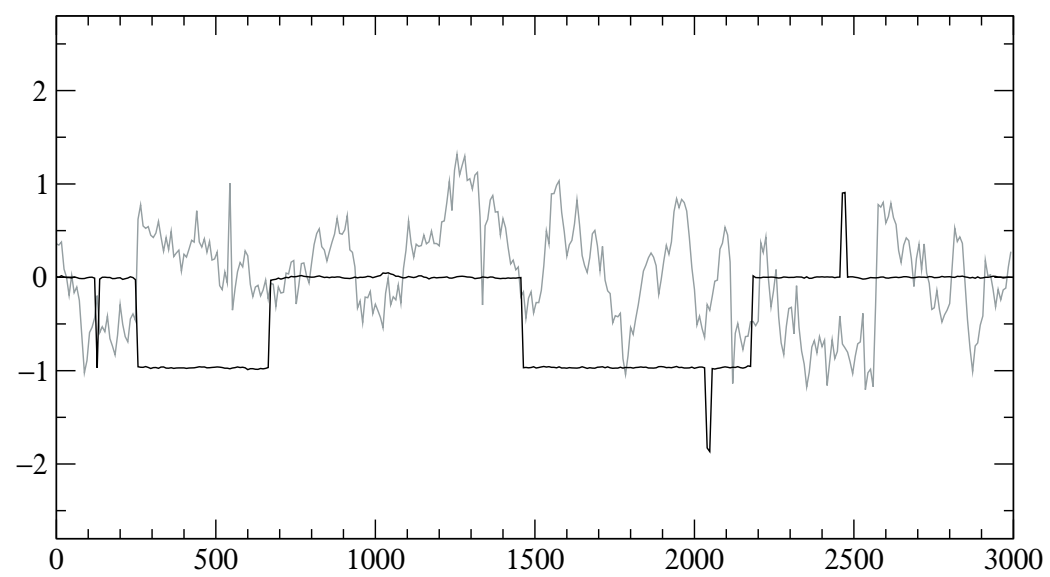

Figure 3. History of the topological charge in three-flavour QCD on a $36 \times 24^{3}$ lattice with SF (black line) and open-SF (grey line) boundary conditions, plotted as a function of the simulation time in units of molecular-dynamics time (see subsection 5.2 for further details).

gauge action (2.5) and three flavours of nearly massless quarks was simulated. ${ }^{4}$ All simulations reported in this paper were performed with a version of the HMC algorithm [33], as described in ref. [24] and implemented in the publicly available openQCD program package [34].

At the chosen point in parameter space, the spatial size $L$ of the lattice is about $1.2 \mathrm{fm}$ [31]. The lattice considered is thus representative of the situation typically encountered at the low-energy end of a step-scaling iteration, where at most one or two further steps need to be taken before contact with the large-volume regime of the theory can safely be made. As is evident from figure 3, the topological charge sectors are poorly sampled in this case. Moreover, when the lattice spacing is reduced at fixed $L$, the associated autocorrelation times grow very rapidly and the simulation algorithm will, in practice, be trapped in a single charge sector.

The loss of ergodicity of the simulations compromises their correctness and must be expected to affect the calculated quantities in various ways. Provided the simulation algorithm gets trapped in the charge zero sector, the issue may be less relevant on lattices with sizes $T, L \ll 1 \mathrm{fm}$, where the QCD functional integral is totally dominated by the gauge fields in a neighborhood of the classical vacuum configuration. In the continuum theory and at fixed $T / L$, the variance $\left\langle Q^{2}\right\rangle$ of the topological charge is expected to be a universal function of the gradient-flow coupling, which decreases roughly like

$$
\left\langle Q^{2}\right\rangle \propto \bar{g}^{2 \nu} \mathrm{e}^{-b / \bar{g}^{2}}
$$

with $L$. The simulation data for the variance shown in figure 4 actually reach fairly small values already at $L \simeq 1 \mathrm{fm}$, but for the charge fluctuations to be completely negligible,

\footnotetext{
${ }^{4}$ The parameters of the gauge action were $\beta=3.81, c_{0}=5 / 3$ and $c_{1}=-1 / 12$. For the coefficient of the Sheikholeslami-Wohlert term in the quark action, the non-perturbatively determined value $c_{\mathrm{sw}}=$ 1.635109 [31] was used. The coefficients of the $\mathrm{O}(a)$ boundary counterterms were all set to their tree-level values. At the chosen value of the quark hopping parameter, $\kappa=0.137119$, the current-quark masses are practically equal to zero on this lattice [32].
} 


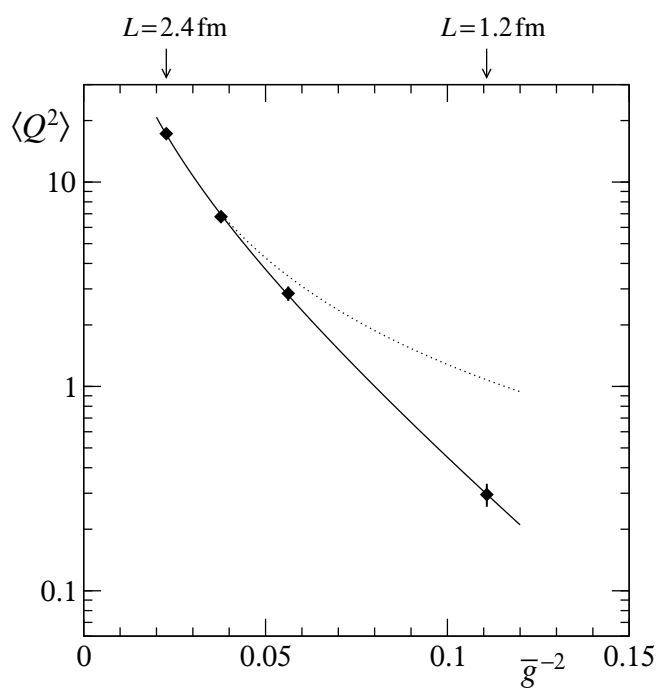

Figure 4. Variance of the topological charge $Q$ (data points) in the SU(3) gauge theory on a $24^{4}$ lattice with SF boundary conditions, plotted as a function of the inverse of the gradient-flow coupling (4.11) with $c$ set to 0.3. The full line is a fit to the data of the form (5.2), while the dotted line indicates where the data would have to lie if the susceptibility $\left\langle Q^{2}\right\rangle / L^{4}$ were independent of the volume.

the box size may have to be as small as $0.3 \mathrm{fm}$ or even smaller. In practice, step-scaling iterations thus necessarily pass through at least a few values of $L$, where the contributions of the non-trivial charge sectors and the topology-freezing issue cannot be ignored.

\subsection{Autocorrelation times from large to small volumes}

With open-SF boundary conditions, the topological charge is not quantized and the field space remains connected in the continuum limit. There is therefore no reason to expect the simulations to become effectively non-ergodic when the lattice spacing is taken to zero. Whether the change from SF to open-SF boundary conditions is profitable in practice however depends on whether the relevant autocorrelation times are significantly reduced or not.

In order to ensure a fair comparison, the summation range in the definition (5.1) of the topological charge on the lattices with open-SF boundary conditions is chosen so as to maximize the integrated autocorrelation times of $Q$ and thus excludes the time-slices near the open boundary, where the charge density fluctuates more rapidly than elsewhere in the space-time volume $[19,35]$. The integrated autocorrelation time of $Q$ then turns out to be larger than the one of all other observables considered and is probably close to the leading exponential autocorrelation time.

In the case of the three-flavour theory considered at the beginning of subsection 5.2, the beneficial effect of choosing open-SF boundary conditions is evident (see figure 3). Reliable determinations of autocorrelation times however require much longer runs than reported in figure 3. The results of some extensive simulations of the $\mathrm{SU}(3)$ gauge theory with Wilson plaquette action are listed in table 1 . While the lattice size was held fixed in these calculations, the inverse gauge coupling $\beta=6 / g_{0}^{2}$ and thus the box size $L$ in physical units 


\begin{tabular}{|c|cccc|cccc|}
\hline & \multicolumn{5}{|c|}{$\mathrm{SF}$} & \multicolumn{4}{c|}{ open-SF } \\
\cline { 2 - 8 }$\beta$ & $\bar{g}^{2}$ & $\tau_{\text {int }}(Q)$ & $\tau_{\text {int }}\left(Q^{2}\right)$ & $\tau_{\text {int }}(\bar{E})$ & $\bar{g}^{2}$ & $\tau_{\text {int }}(Q)$ & $\tau_{\text {int }}\left(Q^{2}\right)$ & $\tau_{\text {int }}(\bar{E})$ \\
\hline 5.96 & $44.1(2)$ & $49(6)$ & $25(2)$ & $25(2)$ & $39.9(2)$ & $51(7)$ & $30(4)$ & $24(2)$ \\
6.09 & $26.5(1)$ & $154(22)$ & $70(8)$ & $46(5)$ & $24.2(1)$ & $152(29)$ & $79(12)$ & $45(6)$ \\
6.21 & $17.8(1)$ & $386(51)$ & $189(24)$ & $74(8)$ & $16.5(1)$ & $297(47)$ & $138(12)$ & $62(6)$ \\
6.42 & $9.0(1)$ & $1595(199)$ & $1138(162)$ & $315(40)$ & $9.63(2)$ & $438(66)$ & $199(19)$ & $93(7)$ \\
6.59 & & & & & $6.92(3)$ & $176(23)$ & $125(14)$ & $74(8)$ \\
7.00 & & & & & $4.21(2)$ & $58(7)$ & $35(4)$ & $31(3)$ \\
7.50 & & & & & $2.97(1)$ & $31(2)$ & $23(1)$ & $26(2)$ \\
30.00 & & & & & $0.24(1)$ & $6(1)$ & $4(1)$ & $3(1)$ \\
\hline
\end{tabular}

Table 1. Integrated autocorrelation times in molecular-dynamics units on a $24^{4}$ lattice.

varies over a range of values. Apart from the integrated autocorrelation times of $Q, Q^{2}$ and

$$
\bar{E}=\left.L^{-3} \sum_{\mathbf{x}} E(t, x)\right|_{x_{0}=L / 2}
$$

the gradient-flow coupling is quoted for both SF and open-SF boundary conditions. All observables were evaluated at flow time $t$ corresponding to $\sqrt{8 t} / L=0.3$.

In the range of $\beta$ from 5.96 to 6.42 , the box size $L$ decreases from about 2.4 to $1.2 \mathrm{fm}$. All autocorrelation times listed in table 1 grow rapidly in this range, particularly so in the case of SF boundary conditions. At smaller values of $L$, simulations of the Schrödinger functional tend to be trapped in a sector of fixed topological charge and the determination of the autocorrelation times becomes impractical. With open-SF boundary conditions, on the other hand, the simulations do not show any sign of a freezing effect. Moreover, towards the perturbative regime, the calculated autocorrelation times decrease and reach fairly small values at $\bar{g}^{2} \leq 3$.

\subsection{Scaling behaviour and statistical errors}

The autocorrelation times quoted in table 1 were measured on a fixed lattice. If the gradient-flow coupling is held fixed instead, the autocorrelation times must be expected to grow with the lattice size. They tend to increase very rapidly in the case of SF boundary conditions as soon as the topology-freezing effects set in [26-28]. With open-SF boundary conditions, the autocorrelation times probably grow approximately like $1 / L^{2}$ [19], except perhaps at very small coupling, where the theory is nearly Gaussian and the autocorrelation times may conceivably grow more slowly [36].

Since the variance of the gradient-flow coupling is a renormalized quantity of order $\bar{g}^{4}$, the number of statistically independent measurements of the coupling required for a given relative statistical error is expected to be practically independent of the lattice spacing and only slowly varying with the physical size of the lattice. The empirical studies reported in this section confirm this and moreover show that, with only 100 measurements, the values 
of the coupling quoted in table 1 could be reproduced to a precision of about $1 \%(0.6 \%$ deep in the perturbative regime).

\section{Boundary lattice effects}

Boundary lattice effects decrease only linearly with the lattice spacing and are therefore potentially large. By tuning the coefficients of the appropriate boundary counterterms, they can in principle be reduced to $\mathrm{O}\left(a^{2}\right)$, but so far this has only been possible at low orders of perturbation theory.

As explained below, the expectation values of observables localized in the center of the lattice tend to be weakly affected by the boundary lattice effects. In practice, and if only such observables are considered, a fine-tuning of the improvement coefficients is then unnecessary.

\subsection{How large are the boundary lattice effects?}

A first impression of how important the boundary lattice effects are can be obtained by studying the dependence of the quantities of interest on the boundary improvement coefficients. The data plotted in figure 5, for example, show that the gradient-flow coupling depends only weakly on $c_{\mathrm{G}}$ and $c_{\mathrm{G}}^{\prime}$ at the points in parameter space considered. On these lattices, and if the values of the coefficients where $\mathrm{O}(a)$-improvement is achieved are in the range $[0.5,1.5]$, the boundary lattice effects at $c_{\mathrm{G}}=c_{\mathrm{G}}^{\prime}=1$ are thus at most one percent or so.

Deep in the perturbative regime, the improvement coefficients are close to their values at tree-level of perturbation theory. In the $\mathrm{O}(a)$-improved theory with $\mathrm{SU}(3)$ gauge group, Wilson gauge action and $N_{\mathrm{f}}$ quark flavours, for example, the one-loop correction in

$$
c_{\mathrm{G}}^{\prime}=1+\left\{-0.08900(5)+0.0191410(1) \times N_{\mathrm{f}}\right\} g_{0}^{2}+\mathrm{O}\left(g_{0}^{4}\right)
$$

is indeed reasonably small $[37,38]$. The gradient-flow coupling (at, say, $c \leq 0.3$ and $L \geq 16)$ is therefore expected to be affected by $\mathrm{O}(a)$ boundary effects at a level of at most a small fraction of a percent in this regime if the improvement coefficients are set to their tree-level values. Clearly, the situation may be less favourable at large couplings and nonperturbative scaling studies are required to be able to exclude the presence of significant residual $\mathrm{O}(a)$ effects (see subsection 6.3).

\subsection{Finite-size effects and the large-volume limit}

The simulation results shown in figure 5 can be theoretically understood, to some extent at least, by studying the volume dependence of the expectation values of observables $\mathcal{O}(x)$ localized in the center of the space-time volume. In the present context, the observable of interest is $E(t, x)$ at some flow-time $t$, but the argumentation in the following paragraphs applies to any observable $\mathcal{O}(x)$ with vacuum quantum numbers and fixed localization range.

Finite-volume effects are universal and are best discussed directly in the continuum theory. Clearly, differentiation of $\langle\mathcal{O}(x)\rangle$ with respect to $T$ amounts to inserting the Euclidean expression for the Hamilton operator in the expectation value. At fixed $T / L$, the 

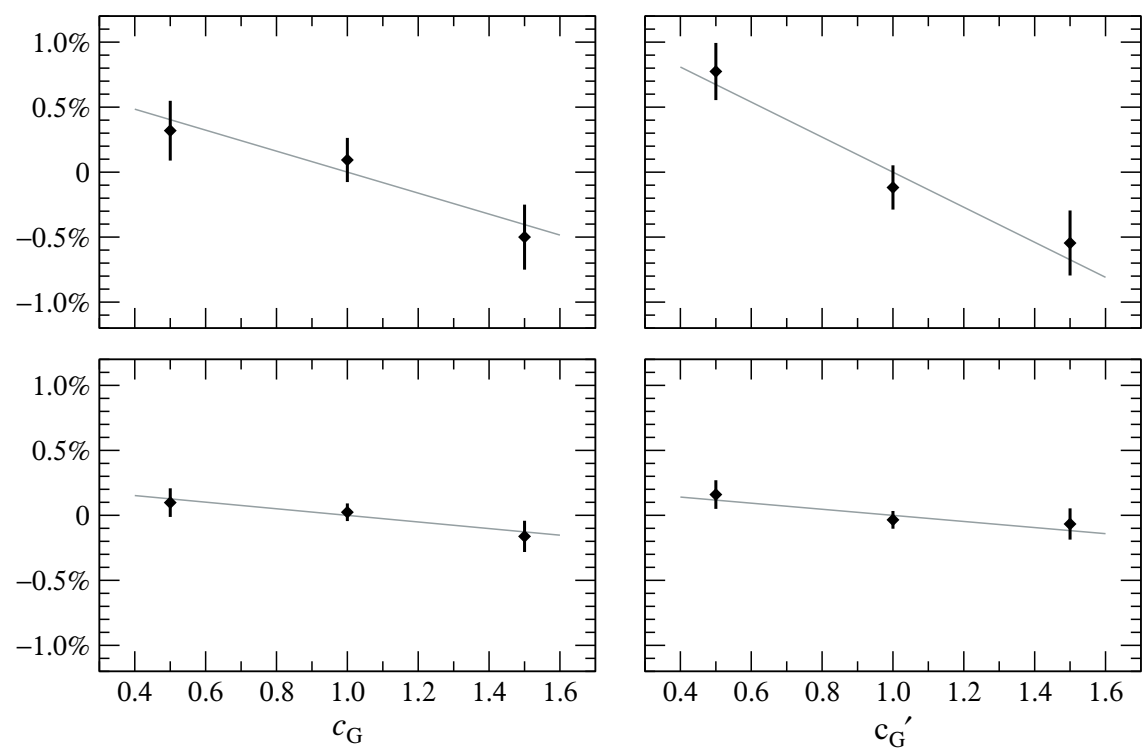

Figure 5. Sensitivity of the gradient-flow coupling $\bar{g}^{2}$ to changes of the improvement coefficients $c_{\mathrm{G}}$ (left column) and $c_{\mathrm{G}}^{\prime}$ (right column) in the $\mathrm{SU}(3)$ gauge theory with Wilson action and open-SF boundary conditions. All data points were obtained on $24^{4}$ lattices at $\beta=7.5$ and are normalized so that the fitted straight lines pass through zero at $c_{\mathrm{G}}=c_{\mathrm{G}}^{\prime}=1$. In the upper and lower rows of plots, the coefficient $c$ [eq. (4.11)] was set to 0.3 and 0.2 , respectively. The associated central values of the coupling are 2.97 and 2.53 .

differential relation

$$
\frac{\partial\langle\mathcal{O}(x)\rangle}{\partial L}=\frac{1}{2} \sum_{\mathbf{y}}\left\langle\mathcal{O}(x)\left\{\left.T_{00}(y)\right|_{y_{0}=0}+\left.T_{00}(y)\right|_{y_{0}=T}\right\}\right\rangle_{\mathrm{c}}+\ldots
$$

is thus obtained, where $T_{\mu \nu}(y)$ denotes the energy-momentum tensor and the ellipsis stands for three further terms in which one of the space coordinates plays the rôle of time. As indicated by the subscript "c", the two-point functions in this formula are the connected parts of the full correlation functions.

When probed by local fields at distances significantly larger than its localization range, as is the case in eq. (6.2) if $L$ is large, the observable $\mathcal{O}(x)$ behaves like a strictly local field of dimension $d \geq 4[3,15]$. Moreover, in the perturbative regime of QCD, the dilation symmetry is only broken by logarithms of the scale factor (as before, the quark masses are assumed to vanish). The $L$-dependent parts of $\langle\mathcal{O}(x)\rangle$ are therefore expected to fall off approximately like $L^{-d}$ at large $L$ until the large-volume regime of the theory is reached, where scale invariance is strongly violated. At this point, the remaining finite-volume effects however tend to be already much smaller than $\langle\mathcal{O}(x)\rangle$ to the extent that they can usually be neglected. ${ }^{5}$

Numerical simulations confirm that the expectation value of $E(t, x)$ converges to its infinite-volume limit in this way (see figure 6). In the range $L \geq 4 \sqrt{8 t}$, and within statistical

\footnotetext{
${ }^{5}$ In the theory with two or more flavours of massless quarks, the asymptotic form of the finite-volume effects can be worked out in chiral perturbation theory [39]. The leading terms are of order $L^{-6}$ if periodic boundary conditions are chosen and otherwise of order $L^{-4}$.
} 


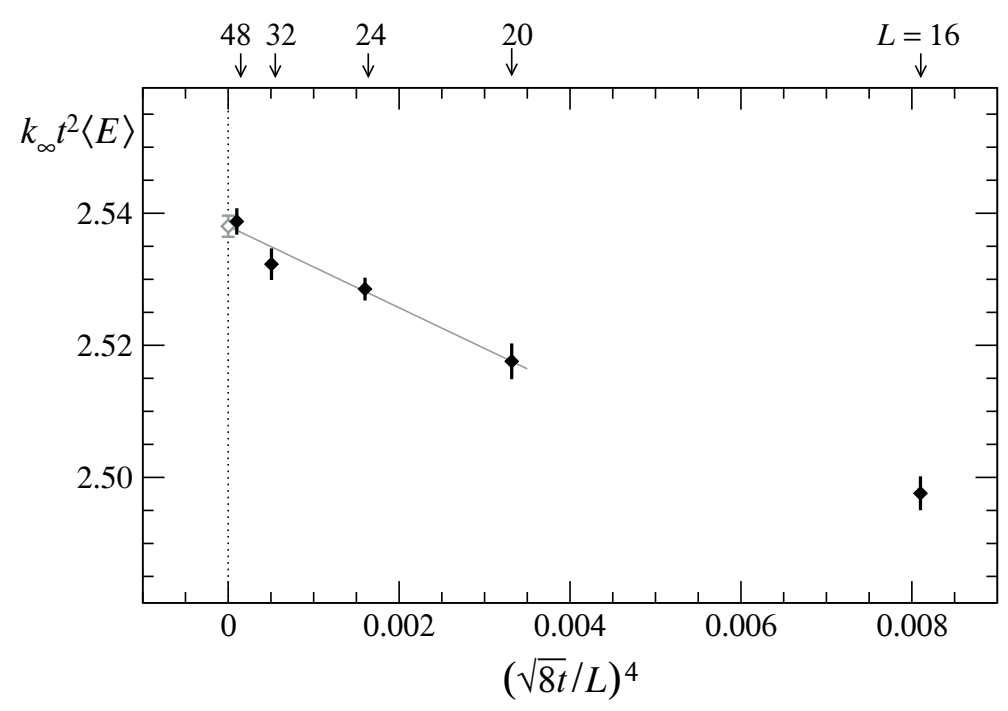

Figure 6. Volume dependence of $k_{\infty} t^{2}\langle E(t, x)\rangle$ at $x_{0}=L / 2$ and fixed $t$. The black data points were obtained by simulating the $\mathrm{SU}(3)$ gauge theory at $\beta=7.5$ on a range of $L^{4}$ lattices with open-SF boundary conditions and tree-level improved Wilson gauge action. The chosen value of the flow time $t$ is such that $\sqrt{8 t} / L=0.3$ and $\bar{g}^{2}=2.501(3)$ at $L=16$.

errors of about one per mille, the data plotted in figure 6 lie on a straight line that extrapolates to $\bar{g}_{\infty}^{2}=2.538(2)$ (grey line and data point). Moreover, even on the smallest lattice considered, the expectation value differs from its infinite-volume limit by less than $2 \%$.

\subsection{Synthesis}

The volume dependence of the expectation values $\langle\mathcal{O}(x)\rangle$ and their dependence on the improvement coefficients $c_{\mathrm{G}}$ and $c_{\mathrm{G}}^{\prime}$ are closely related to each other. Differentiation of $\langle\mathcal{O}(x)\rangle$ with respect to the latter actually leads to expressions like the one in eq. (6.2). The derivatives are therefore expected to be of order $1 / L^{5}$ in the perturbative regime. In particular, the contribution of the $\mathrm{O}(a)$ boundary counterterms to the gradient-flow coupling is proportional to $t^{2} / L^{5}$, which explains the difference (by about a factor 5 ) of the data in the upper and lower row of plots in figure 5.

Eventually, the importance of the $\mathrm{O}(a)$ boundary effects should be assessed by performing suitable scaling tests. The step-scaling function $\Sigma(s, u, 1 / L)$ of the gradient-flow coupling, for example, is expected to approach its continuum limit with a rate proportional to $1 / L^{2}$ (rather than $\left.1 / L\right)$ if there are no significant $\mathrm{O}(a)$ effects $[1,37]$. Consistently with the theoretical discussion in this section and the reported empirical results, the data shown in figure 7 are in fact compatible with the residual $\mathrm{O}(a)$ boundary effects being smaller than the statistical errors.

In practice the choice of the parameter $c=\sqrt{8 t} / L$ of the gradient-flow coupling may require some tuning. At small values of $c$, the $\mathrm{O}(a)$ boundary effects are strongly suppressed, but the smoothing range $\sqrt{8 t}$ must also be significantly larger than the lattice spacing in order to avoid large $\mathrm{O}\left(a^{2}\right)$ contributions to the expectation value of $E(t, x)$. Increasing $T / L$ may be worth considering if these criteria cannot both be met on the accessible lattices. 


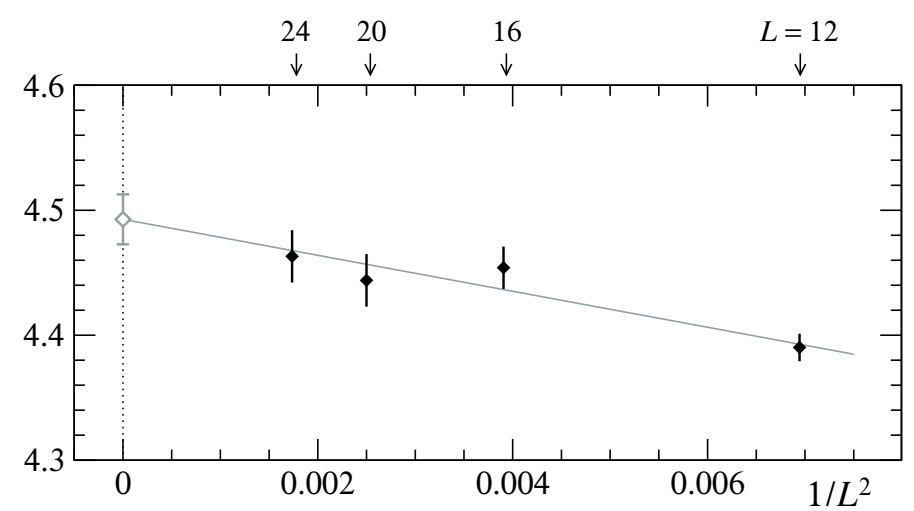

Figure 7. Dependence on the lattice spacing of the step-scaling function $\Sigma(2, u, 1 / L)$ in the treelevel $\mathrm{O}(a)$-improved $\mathrm{SU}(3)$ gauge theory at $u=2.968$ (black data points). In this calculation, the Wilson gauge action was used and the coefficient $c$ [eq. (4.11)] was set to 0.3. A linear extrapolation of the plotted data yields the value $\sigma(2, u)=4.49(2)$ for the step-scaling function in the continuum theory (grey data point).

\section{Concluding remarks}

The combination of observables and boundary conditions advertised in this paper provides a technically attractive framework for step-scaling studies. Since the topology-freezing issue is avoided, non-perturbative computations of Symanzik improvement coefficients (such as those recently reported in [31, 32]) are likely to profit from the use of open-SF boundary conditions as well. The fact that the gauge and ghost propagators assume a simple form moreover suggests that numerical stochastic perturbation theory is straightforward to implement with these boundary conditions (see ref. [40] for a review of numerical stochastic perturbation theory and $[41,42]$ for the latest advances in this field).

In QCD many observables suitable for step scaling can be constructed using the extension of the Yang-Mills gradient flow to the quark fields [4]. Unlike the case of periodic boundary conditions [43], the chosen observables should preferably be localized in the central region of the space-time volume as otherwise the $\mathrm{O}(a)$-improvement of the calculated quantities may be complicated by boundary lattice effects. The normalization of the renormalized quark field $\chi_{\mathrm{R}}(t, x)$ at flow-time $t>0$, for example, may be fixed by requiring the expectation value of the observable

$$
\mathcal{O}(x)=\bar{\chi}_{\mathrm{R}}(t, x)(\not D-\overleftarrow{\not D}) \chi_{\mathrm{R}}(t, x)
$$

at $x_{0}=L / 2$ to be equal to its value at tree-level of perturbation theory [14]. Local fields at vanishing flow time can then be renormalized by probing them with suitable products of renormalized fields at $t>0$.

The fact that the gradient-flow coupling $\bar{g}_{\infty}^{2}$ in infinite volume can be accurately computed at all flow times visited in the course of a step-scaling calculation is intriguing. Extrapolations to the continuum and the infinite-volume limit are required in this computation, but both limits are reached fairly rapidly at fixed flow time and could actually be taken simultaneously. Current correlation functions in position space and other quantities 
of interest might be accessible in this way too, provided they depend on a single external scale that can play the rôle of the flow time.

\section{Acknowledgments}

I am indebted to John Bulava for helpful correspondence on three-flavour QCD with SF boundary conditions and the topology-freezing issue in this theory. Thanks also go to Agostino Patella and Stefan Schaefer for interesting discussions on various topics addressed in this paper. All simulations were performed on a dedicated PC cluster at CERN. I am grateful to the CERN management for funding this machine and to the CERN IT Department for technical support.

\section{A Notational conventions}

The gauge group is taken to be $\mathrm{SU}(N)$ with $N=3$ in sections 5 and 6 . In any basis $T^{a}$, $a=1, \ldots, N^{2}-1$, of complex, anti-Hermitian and traceless $N \times N$ matrices, the general element of the Lie algebra of $\mathrm{SU}(N)$ is given by $X^{a} T^{a}$ with real components $X^{a}$ (repeated group indices are automatically summed over). The generators are assumed to satisfy

$$
\operatorname{tr}\left\{T^{a} T^{b}\right\}=-\frac{1}{2} \delta^{a b}
$$

but are otherwise left unspecified. Lorentz indices $\mu, \nu, \ldots$ range from 0 to 3 and are not automatically summed over when they occur in matching pairs.

The differential operators $\partial_{x, \mu}^{a}$ act on differentiable functions $f(U)$ of the gauge field $U$ according to

$$
\partial_{x, \mu}^{a} f(U)=\left.\frac{\mathrm{d}}{\mathrm{d} s} f\left(\mathrm{e}^{s X} U\right)\right|_{s=0}, \quad X(y, \nu)= \begin{cases}T^{a} & \text { if }(y, \nu)=(x, \mu), \\ 0 & \text { otherwise. }\end{cases}
$$

While these operators depend on the choice of the generators $T^{a}$, the gradient field $T^{a} \partial_{x, \mu}^{a} f(U)$ can be shown to be basis-independent.

\section{B Normalization of the gradient-flow coupling}

The computation of the normalization factor

$$
k^{-1}=\left\{t^{2} \mathcal{E}_{0}(t, x)\right\}_{T=L, x_{0}=L / 2, \sqrt{8 t}=c L}
$$

in eq. (4.11) requires the expectation value

$$
\langle E(t, x)\rangle=\mathcal{E}_{0}(t, x) g_{0}^{2}+\mathrm{O}\left(g_{0}^{4}\right)
$$

to be calculated at tree-level of perturbation theory. In the lattice theory with the Wilson plaquette action and SF boundary conditions, the calculation was recently performed by Fritzsch and Ramos $[7,8]$. The results quoted below hold for all actions considered in this paper and cover the case of both open-SF and SF boundary conditions. 


\section{B.1 Open-SF boundary conditions}

To leading order in the gauge coupling, $E(t, x)$ is a quadratic expression in the gauge potential at flow time $t$ and thus, via eq. (4.7), of the fundamental field. Recalling eq. (3.17), the result

$$
\mathcal{E}_{0}(t, x)=\frac{N^{2}-1}{T L^{3}} \sum_{p} \mathrm{e}^{-2 t \hat{p}^{2}}\left\{\sin \left(p_{0} x_{0}\right)^{2} \sum_{l=1}^{3} S_{l 0}(p)+\cos \left(p_{0} x_{0}\right)^{2} \sum_{l>j=1}^{3} S_{l j}(p)\right\}
$$

is then obtained, where

$$
S_{\mu \nu}(p)=\left(1-\frac{1}{4} \hat{p}_{\mu}^{2}\right)\left(1-\frac{1}{4} \hat{p}_{\nu}^{2}\right)\left\{\hat{p}_{\mu}^{2} D_{\nu \nu}(p)+\hat{p}_{\nu}^{2} D_{\mu \mu}(p)-2 \hat{p}_{\mu} \hat{p}_{\nu} D_{\mu \nu}(p)\right\} .
$$

Substitution of these equations in eq. (B.1) yields the normalization factor in the form of a momentum sum that can be evaluated numerically.

\section{B.2 SF boundary conditions}

For these boundary conditions, the normalization factor is again given by eq. (B.1), but the expression for

$$
\mathcal{E}_{0}(t, x)=\frac{N^{2}-1}{T L^{3}} \sum_{p}^{\prime} \mathrm{e}^{-2 t \hat{p}^{2}}\left\{\cos \left(p_{0} x_{0}\right)^{2} \sum_{l=1}^{3} S_{l 0}(p)+\sin \left(p_{0} x_{0}\right)^{2} \sum_{l>j=1}^{3} S_{l j}(p)\right\}
$$

differs from the one obtained in the case of open-SF boundary conditions. In particular, the sines and cosines in the curly bracket are interchanged with respect to eq. (B.3) and the time component of the momentum,

$$
p_{0}=n_{0} \frac{\pi}{T}, \quad n_{0}=0,1, \ldots, T-1,
$$

is summed over a different set of values. The primed summation symbol in eq. (B.5) indicates that the terms at $p_{0}=0$ are given the weight $1 / 2$ and that the $p=0$ term is to be omitted.

\section{B.3 Explicit form of the gauge-field propagator}

Equation (B.4) involves the gauge-field propagator $D_{\mu \nu}(p)$ and is therefore not fully explicit. Starting from the inverse propagator (3.15), a few lines of algebra however show that

$$
D_{\mu \nu}(p)=\frac{\delta_{\mu \nu}}{r_{\mu}}-\frac{v_{\mu} v_{\nu}}{1+\sum_{\rho} v_{\rho}^{2} r_{\rho}}+\left(\lambda_{0}^{-1}-1\right) \frac{\hat{p}_{\mu} \hat{p}_{\nu}}{\left(\hat{p}^{2}-c_{1} \hat{p}^{4}\right)^{2}},
$$

where

$$
r_{\mu}=\hat{p}^{2}-c_{1}\left(\hat{p}^{4}+\hat{p}^{2} \hat{p}_{\mu}^{2}\right), \quad v_{\mu}=c_{1} \hat{p}_{\mu}^{3} / r_{\mu} .
$$

Note that the second term in eq. (B.7) is of order $a^{4}$ in the lattice spacing, while the last term is a gauge term that cancels in eq. (B.4). 
Open Access. This article is distributed under the terms of the Creative Commons Attribution License (CC-BY 4.0), which permits any use, distribution and reproduction in any medium, provided the original author(s) and source are credited.

\section{References}

[1] M. Lüscher, P. Weisz and U. Wolff, A numerical method to compute the running coupling in asymptotically free theories, Nucl. Phys. B 359 (1991) 221 [INSPIRE].

[2] M. Lüscher, Properties and uses of the Wilson flow in lattice QCD, JHEP 08 (2010) 071 [Erratum ibid. 03 (2014) 092] [arXiv: 1006 .4518] [INSPIRE].

[3] M. Lüscher and P. Weisz, Perturbative analysis of the gradient flow in non-Abelian gauge theories, JHEP 02 (2011) 051 [arXiv: 1101.0963] [INSPIRE].

[4] M. Lüscher, Chiral symmetry and the Yang-Mills gradient flow, JHEP 04 (2013) 123 [arXiv: 1302.5246] [INSPIRE].

[5] Z. Fodor, K. Holland, J. Kuti, D. Nogradi and C.H. Wong, The Yang-Mills gradient flow in finite volume, JHEP 11 (2012) 007 [arXiv: 1208.1051] [INSPIRE].

[6] Z. Fodor, K. Holland, J. Kuti, D. Nogradi and C.H. Wong, The gradient flow running coupling scheme, PoS (LATTICE 2012) 050 [arXiv:1211.3247] [INSPIRE].

[7] P. Fritzsch and A. Ramos, The gradient flow coupling in the Schrödinger functional, JHEP 10 (2013) 008 [arXiv: 1301.4388] [INSPIRE].

[8] P. Fritzsch and A. Ramos, Studying the gradient flow coupling in the Schrödinger functional, PoS(LATTICE 2013) 319 [arXiv: 1308.4559] [INSPIRE].

[9] A. Ramos, The gradient flow in a twisted box, PoS (LATTICE 2013) 053 [arXiv:1308.4558] [INSPIRE].

[10] J. Rantaharju, The gradient flow coupling in minimal walking technicolor, PoS (LATTICE 2013) 084 [arXiv: 1311.3719] [INSPIRE].

[11] H. Suzuki, Energy-momentum tensor from the Yang-Mills gradient flow, Prog. Theor. Exp. Phys. 2013 (2013) 083B03 [arXiv: 1304.0533] [INSPIRE].

[12] L. Del Debbio, A. Patella and A. Rago, Space-time symmetries and the Yang-Mills gradient flow, JHEP 11 (2013) 212 [arXiv: 1306.1173] [INSPIRE].

[13] FlowQCD collaboration, M. Asakawa, T. Hatsuda, E. Itou, M. Kitazawa and H. Suzuki, Thermodynamics of SU(3) gauge theory from gradient flow, arXiv:1312.7492 [INSPIRE].

[14] H. Makino and H. Suzuki, Lattice energy-momentum tensor from the Yang-Mills gradient flow - inclusion of fermion fields, Prog. Theor. Exp. Phys. 2014 (2014) 063B02 [arXiv: 1403.4772] [INSPIRE].

[15] M. Lüscher, Future applications of the Yang-Mills gradient flow in lattice QCD, PoS (LATTICE 2013) 016 [arXiv: 1308.5598] [INSPIRE].

[16] M. Lüscher, R. Narayanan, P. Weisz and U. Wolff, The Schrödinger functional: a renormalizable probe for non-Abelian gauge theories, Nucl. Phys. B 384 (1992) 168 [hep-lat/9207009] [INSPIRE].

[17] S. Sint, On the Schrödinger functional in QCD, Nucl. Phys. B 421 (1994) 135 [hep-lat/9312079] [INSPIRE]. 
[18] P. Fritzsch, A. Ramos and F. Stollenwerk, Critical slowing down and the gradient flow coupling in the Schrödinger functional, PoS (LATTICE 2013) 461 [arXiv:1311.7304] [INSPIRE].

[19] M. Lüscher and S. Schaefer, Lattice QCD without topology barriers, JHEP 07 (2011) 036 [arXiv: 1105.4749] [INSPIRE].

[20] K.G. Wilson, Confinement of quarks, Phys. Rev. D 10 (1974) 2445 [inSPIRE].

[21] Y. Iwasaki, Renormalization group analysis of lattice theories and improved lattice action. II. Four-dimensional non-Abelian $\mathrm{SU}(N)$ gauge model, preprint UTHEP-118, (1983) [arXiv: 1111.7054] [INSPIRE].

[22] M. Lüscher and P. Weisz, On-shell improved lattice gauge theories, Commun. Math. Phys. 97 (1985) 59 [Erratum ibid. 98 (1985) 433] [INSPIRE].

[23] S. Aoki, R. Frezzotti and P. Weisz, Computation of the improvement coefficient $c_{\mathrm{sw}}$ to one loop with improved gluon actions, Nucl. Phys. B 540 (1999) 501 [hep-lat/9808007] [INSPIRE].

[24] M. Lüscher and S. Schaefer, Lattice QCD with open boundary conditions and twisted-mass reweighting, Comput. Phys. Commun. 184 (2013) 519 [arXiv:1206.2809] [INSPIRE].

[25] M. Bruno and R. Sommer, On the $N_{f}$-dependence of gluonic observables, PoS (LATTICE 2013) 321 [arXiv: 1311.5585] [INSPIRE].

[26] L. Del Debbio, H. Panagopoulos and E. Vicari, $\theta$-dependence of $\mathrm{SU}(N)$ gauge theories, JHEP 08 (2002) 044 [hep-th/0204125] [INSPIRE].

[27] S. Schaefer, R. Sommer and F. Virotta, Investigating the critical slowing down of QCD simulations, PoS (LAT2009) 032 [arXiv: 0910.1465] [INSPIRE].

[28] ALPHA collaboration, S. Schaefer, R. Sommer and F. Virotta, Critical slowing down and error analysis in lattice QCD simulations, Nucl. Phys. B 845 (2011) 93 [arXiv:1009.5228] [INSPIRE].

[29] B. Sheikholeslami and R. Wohlert, Improved continuum limit lattice action for QCD with Wilson fermions, Nucl. Phys. B 259 (1985) 572 [InSPIRE].

[30] M. Lüscher, S. Sint, R. Sommer and P. Weisz, Chiral symmetry and $O(a)$ improvement in lattice QCD, Nucl. Phys. B 478 (1996) 365 [hep-lat/9605038] [INSPIRE].

[31] J. Bulava and S. Schaefer, Improvement of $N_{f}=3$ lattice QCD with Wilson fermions and tree-level improved gauge action, Nucl. Phys. B 874 (2013) 188 [arXiv:1304.7093] [INSPIRE].

[32] J. Bulava, M. Della Morte, J. Heitger and C. Wittemeier, Determination of $c_{\mathrm{A}}$ in three-flavour lattice QCD with Wilson fermions and tree-level improved gauge action, PoS(LATTICE 2013) 311 [arXiv: 1312.3591] [INSPIRE].

[33] S. Duane, A.D. Kennedy, B.J. Pendleton and D. Roweth, Hybrid Monte Carlo, Phys. Lett. B 195 (1987) 216 [INSPIRE].

[34] OpenQCD: simulation program for lattice QCD, http://cern.ch/luscher/openQCD.

[35] G. McGlynn and R.D. Mawhinney, Scaling, topological tunneling and actions for weak coupling DWF calculations, PoS(LATTICE 2013) 027 [arXiv:1311.3695] [INSPIRE].

[36] M. Lüscher and S. Schaefer, Non-renormalizability of the HMC algorithm, JHEP 04 (2011) 104 [arXiv: 1103.1810] [INSPIRE]. 
[37] M. Lüscher, R. Sommer, P. Weisz and U. Wolff, A precise determination of the running coupling in the SU(3) Yang-Mills theory, Nucl. Phys. B 413 (1994) 481 [hep-lat/9309005] [INSPIRE].

[38] S. Sint and R. Sommer, The running coupling from the QCD Schrödinger functional: a one loop analysis, Nucl. Phys. B 465 (1996) 71 [hep-lat/9508012] [INSPIRE].

[39] O. Bär and M. Golterman, Chiral perturbation theory for gradient flow observables, Phys. Rev. D 89 (2014) 034505 [arXiv: 1312.4999] [InSPIRE].

[40] F. Di Renzo and L. Scorzato, Numerical stochastic perturbation theory for full QCD, JHEP 10 (2004) 073 [hep-lat/0410010] [INSPIRE].

[41] M. Brambilla, M. Dalla Brida, F. Di Renzo, D. Hesse and S. Sint, Numerical stochastic perturbation theory in the Schrödinger functional, PoS (LATTICE 2013) 325 [arXiv: 1310.8536] [INSPIRE].

[42] M. Dalla Brida and D. Hesse, Numerical stochastic perturbation theory and the gradient flow, PoS(LATTICE 2013) 326 [arXiv: 1311.3936] [INSPIRE].

[43] C. Monahan and K. Orginos, Finite volume renormalization scheme for fermionic operators, PoS (LATTICE 2013) 443 [arXiv: 1311.2310] [INSPIRE]. 